bowels. I always observe, that those patients whose mouths can be made sore, recover; but mercury often fails to produce this effect without bleeding, and it was evident to those who observed the progress of this fever, that the latter remedy, and especially topical bleeding, had by far the most decided effect on the disease. I may remark, that the apothecary, who, beforehand, was by no means inclined to think favourably of venesection in fever, was, during the prevalence of this contagion, so fully persuaded of its good effects, that, being attacked during his unremitting attendance on the sick, by the first symptoms of the fever, viz. severe rigors, headach, sickness, and excessive languor, and being convinced that he was infected, he immediately opened a vein in his own arm, and suffered about two pounds of blood to flow. On the next day he was nearly well.

For the notes of the above cases I am indebted to Mr Morgan, the apothecary to St Peter's Hospital, to whose assiduous and humane attention the poor of this city have been, on the present, and on many former occasions, greatly indebted.

Bristol, May 31, 1817.

\title{
III.
}

On the Mercurial Treatment of Yellow-Fever. By J. B. SHEPPARD, Member of the Royal College of Surgeons in London, and Surgeon in the Royal Navy.

“Obstat quicquid non adjuvat."-QuintiliaN.

7 The various and opposite opinions which have been entertained respecting the nature and origin of the Yellow-Fever, from our earliest acquaintance with the disease, have given rise to a corresponding diversity of treatment, which has proved a constant source of embarrassment to the inexperienced, and of the utmost prejudice in its consequences to the interest of those whose welfare has been involved in the accuracy of the adopted pathology: the notorious contradictions which characterize the different creeds, would seem to preclude the hope of even an approximation of sentiment on this interesting subject. At one period, an erroneous nosological arrangement inculcates a treatment strictly stimulant, which occasionally yields to an opposite pathology, directing an active evacuant system; by others, 
viewed as a specific disease, it is opposed by a supposed specific remedy; while another class, vaccillating between opinions, or desirous of availing themselves of every probable means of success, have recourse to the combination of the two latter practices, from which they anticipate more favourable results than from a solitary adherence to either. All these systems have had their seasons of ascendancy and of disrepute, supplanting each other again and again, in obedience to the predominant doctrines of the day. There is no want of evidence on record of the success of the whole of them; - of their failure we can best judge from the appalling aggregate mortality of the West Indies during the last quarter of a century. To those most conversant with the formidable nature of the disease, the latter circumstance will be less a source of surprise than the various triumphant accounts which daily meet the cye, many of which the more candid and best informed will be disposed to impute, rather to a comparatively mild form of the disease, than to any effect connected with treatment, - an explanation not the most flattering to professional vanity, but which, in truth, however much it may be a subject of regret, ought not to derogate from the value of the art; for although there may be a degree of yellow-fever which bids defiance to the powers of medicine, it is consoling to know, that subordinate forms of the disease more frequently prevail, in which we can take an active and eminently useful part. In proportion to the justice of our views of the nature of the disease, and the energy with which our curative measures are directed, will be the chance of arresting early fever, and of rescuing the patient from a succeeding state of the greatest suffering and risk; while by injudicious treatment the disease may be protracted, and even an originally harmless form of fever converted into one of extreme violence and danger; or, what more frequently happens, the period for doing good may be consumed in the exhibition of inert remedies, and the patient permitted to sink into the grave, without an adequate effort for his preservation. The latter mode of proceeding applies strictly to the exclusive mercurial practice, on which it is at present my object to throw out a few observations.

It may be thought, that the ascendancy which the depletory treatment has of late years acquired in the West Indies, and the reprehension which the indiscriminate mercurial practice has received from time to time from more able pens, might preclucie the necessity of further comment to accomplish its abolition; and $I$ am aware, that to some these remarks may appear to be opposed to a phantom of my own imagination. 
Adverting, however, to the stress which has been laid by some recent writers on the beneficial combination of the mercurial and depletory practices in this and other fevers, by some of whom mercury is still designated as the chief means of cure; and knowing that the exclusive treatment by mercury had still its adherents when I quitted the West Indies a few years since, I am led to believe that the evil, even in its present limited extent, is of sufficient magnitude to warrant a free exposition of its effects, without incurring a just imputation of the offence of supererogation.

In order to appreciate the causes which may have led to the diffusion of the exclusive mercurial treatment of yellow-fever, it will be necessary to glance at the state of the colonies at the period of its introduction. In the year 1798, the remote cause of yellow-fever appears to have existed in most of the West India islands in a high degree of concentration, far exceeding that of many preceding years: the varying states of energy of the endemic cause in different years being cognizable only by their effects, an obvious difficulty accompanies every attempt to investigate the nature of the atmospheric modifications on which such vicissitudes probably depend. I do not profess to enter into such inquiry. It is probable that, in addition to a difference of degree, there may be some unknown change in the nature and constitution of the noxious miasmata ; and, on this point, in explanation of the occasional attacks of the natives and long residents, M. Humboldt remarks, that a very slight variation is sufficient to destroy immunity in those whose organs are become exquisitely sensible of variation from immutable uniformity of meteorological succession; a proposition which he illustrates by reference to Galvanic experiments, which prove that chemical agents excite not only by their quality, but by their order of succession also. The severe aggravation of the endemic in 1793, succeeding a long interval of comparative exemption, was considered to be capable of explanation only by reference to a contagious source, which was supposed to be traced to a new pestilence imported from Africa into the West Indies. Subsequent experience and investigation have shewn the fallacy of this assumption. The scientific traveller above referred to observes, "The opinion that the yellow-fever was imported from the coast of Africa into Grenada, and from thence into Philadelphia, is equally destitute of foundation with the hypothesis, formerly very generally believed, that a squadron from Siam introduced the vomito into America." The truth of this statement of $M$. Humboldt is now very generally acknowledged; and even those who, to express their belief of the contagious 
nature of yellow-fever, inappropriately retain the name which, on that occasion, was conferred on it, in reference to its imputed foreign origin, do not contend for the validity of the African importation.

II. The sensation which the mortality of that period excited, is a proof how much that of former visitations must have been effaced from the recollection, to make it a subject of such surprise and novelty. We are now unfortunately familiar with many paraliel events; and I believe there is a very general concurrence of sentiment, that they are to be ascribed to purely physical and local causes; an opinion which has received abundant corroboration from the absence of contagious properties in the various epidemics which have subsequently prevailed within the tropics, as well as from the unequivocal refutation of the accuracy of the data on which the assumption of importation was originally grounded.

That the germ of yellow-fever was that year developed in an intense degree of force, and possibly with some variation of constitution also, is proved by the non-exemption of even the natives and long residents; and the great mortality which succeeded, and gave rise to the opinion of an imported new pestilence, was the inevitable effect of an unusual accumulation of unassimilated Europeans in an augmented military and naval force, and in merchant-ships collected into convoy masses, in consequence of the recently declared war. The exposure of these men, strangers to the climate, to an exalted power of the noxious cause, is surely an adequate explanation of the vast loss of life which was then sustained, without reference to a contagious origin.

It may not be considered irrelevant to remark, that the events of the last year in the West Indies, in regard to yellow-fever, bear a close analogy to those of 1793 : the political changes consequent to the peace of 1815 , as in the instance of the new war in 1793, had the effect of introducing an increased number of northern strangers into the colonies; the coincidence of a similar aggravation of the intensity of the endemic cause, has produced a similar result. Of the sufferers on the late occasion, the new garrisons of the French islands appear to have formed a large proportion. France having been deprived of the last of her West Indian colonies in 1809 , the troops in her garrisons, after their restoration at the peace, were necessarily strangers to the climate.

To return to the period of 1793 , the public apprehension was naturally commensurate with the mortality; and as credulity generally keeps pace with apprehension, can we be surprised. 
that the doctrine of importation rcceived general and implieit belief?

A new and specific character being given to the endemic, a specific remedy was speedily proposed, and mercury announced as the only medicine capable of arresting its progress. It was held to be incontrovertible evidence of the efficacy of the remedy, that, in those cases where the sensible effects of mercury took place, recovery usually followed; and although the mortality under the new treatment continued to be excessive, yet as the greater number with ptyalism recovered, the failures were not permitted to affect the reputation of the practice.

It is probable, that the observation of the fact of the salivary glands being unintentionally affected, in some cases of recovery, by the calomel combined with the purgatives, may have led to the inference that the declension of fever was the effect of the salivation, whereas the converse of the conclusion is the truth; for, although the two events may appear to be synchronous, yet, as experience proves, that all attempts to excite the specific action of mercury in a system labouring under a high degree of fever are perfectly unavailing, until the fever be moderated by other means, we are justified in concluding, that the declension of fever, and the supervention of salivation, stand in the relation of cause and effect.

The coexistence of febrile and mercurial action is generally adnitted to be incompatible; if therefore the action of mercury could be superinduced in violent fever, we should be possessed of an invaluable remedy; but in a high degree of fever we find mercury to be utterly powerless, in whatever quantity or manner it may be exhibited; and if exclusively confided in, the disease pursues its march unchecked, to disorganization and death, without the appearance of any sensible effect from the mercury employed. On the other hand, if a mitigation of fever takes place, whether spontaneously in the milder cases, or from the employment of more efficient measures in the more severe, then salivation begins; and what is in truth a mere effect, and test of the cessation of fever, and in no degree contributory to the cure, is, by the advocates of mercury, exalted into the sole or chief cause of such salutary event. If, then, in the subordinate forms of yellow-fever, the operation of mercury is found to be superfluous, and in the more severe, its employment is ineffectual, it will be difficult to imagine a case of the legitimate disease in which the adoption of the practice is indispensable; while it must be admitted, that an exclusive reliance on that remedy in all cases of serious import, is not merely futile and deceptive, but replete with the greatest danger. 
The insensibility of the system under active fever to the stimulus of mercury, has been universally observed, and generally referred to derangement of the function of the absorbent in common with other parts of the system during the presence of the morbid actions which constitute fever. When these actions cease, the atsorbent function is restored, and the mercury previously exhibited, which until then had lain dormant, is taken into the system, and its sensible operation rapidly excited; but whatever may be the received opinion of the cause of such insensibility in that condition of the system, the fact itself is palpable, and perfectly conclusive as to the inefficacy of mercury as a remedy in active fever.

It is every day remarked and lamented, that we are so little benefited by the errors and failures of our predecessors, and that our lessons must be dearly purchased in the school of experience, to be deeply imprinted on our recollection. This would be of comparatively little moment, did the consequence devolve upon ourselves only ; but it assumes a widely different aspect and interest, when the lives that are entrusted to us become the price at which wisdom is to be purchased. I cannot therefore sufficiently deprecate a repetition of fruitless attempts to mercurialize the system, to the exclusion of more efficient measures; or sufficiently caution the inexperienced against the vain expectation of achieving what others of the greatest talent and perseverance have failed to effectuate. The consequence is doubly injurious; it is not only the loss to-day, which the industry of to-morrow. may redeem; it too often involves the loss of those monents which cannot be retrieved,- when only the medical art can afford any effectual relief. These reflections are forced from me by a contemplation of the awful amount of mortality that has taken place in the West Indies; and by the painful apprehension, that, on many occasions at least, this might have been diminished by a more efficient practice. It should, however, be recollected, that it was the bounden duty of those on whom the treatment of this disease devolved, at one time, to give every trial to a remedy so (otherwise) potent, and so highly praised; but after it has been so amply tried, and found wanting, the perseverance in a vain experiment is surely incurring no common responsibility : and theretore, it is at least to be hoped, that they who are still inclined to put it to the test, will employ those efficient measures at the same time, which are at once most conducive to its specific effect, and of themselves afford the most reasonable hope of subduing so formidable a disease.

The advantage of the combination of the mercurial with other treatment, I believe, however, to be very questionable. In the 
more rapid and dangerous cases, in which two or three days decide the fate of the patient, (independent of the absolute inertness of mercury in high fever) there is not, in the majority of habits, sufficient time for the operation of the remedy to be excited, betore the disease is either cured by more efficacious means, and salivation becomes worse than useless, or, in the less controllable forms of attack, the patient is irrecoverably lost. Still I admit, that the combined practice does not involve the serious objections which attach to the exclusive dependence on mercury, and am chiefly solicitous to detract from its rank as the principal means of cure. As a cathartic, the employment of calomel in early tever is acknowledged on all hands to be highly beneficial; but from the uncertainty of its operation, where there is much heat or torpor, it should not be trusted to alone, but always be given in conbination with other purgative substances.

With regard to the remedies to which I have adverted, as possessing more efficiency than mercury in the cure of yellowfever, I have nothing new to propose. From the experience of many years within the tropics, I am disposed to coincide with those who believe that the disease, in the highest degree of concentration, is irremediable by any known means in medicine; for I have remarked, in this extreme case, that whatever plan of cure may be adopted, the rate of mortality remained unaffected by variety of treatment. Although this exalted character of the endemic is not rare, when a sufficient number of proper subjects is accumulated, yet, comparatively speaking, it may be deemed unfrequent when contrasted with the great number of cases that are classed and cured under the title of Yellow-Fever. In the minor degrees of violence of this fever, there is perhaps no disease in which the valuable powers of medicine are more decidedly displayed. The treatment may be comprised in a few words. The disease exhibiting violently inordinate actions in the beginning of the attack, early and commensurate depletion, copious purging, with the abstraction of superabundant heat, and the interruption of morbid associations by the affusion of cold water on the surface, comprehend the resources in which we may place the best grounded confidence that it may be subdued at an early period of the invasion. Should these remedies fail in obtaining an early mitigation of the symptoms, I cannot say that $\mathbf{I}$ have observed much decided good from medicine in the sub:equent stages of exhaustion. It is our imperative duty, therefore, to direct all our exertions and means to a decisive impression on the system during the first hours, so as to cut short, or greatly to check the march of the fever. I must however here observe, that, though I have always bled decidedly in the early 
stage of yellow-fever, guided rather by effect than by quantity, my experience leads me to doubt that I could have obtained, or that the abstraction of such large quantities of blood would have been admissible, as appear to have been taken in some less dangerous fevers.

I am aware, that there are exceptions to this rule of practice of active depletion; that a form of fever is occasionally met with in that climate, in which, from modification of cause, or of habit, the vital functions are, from the first invasion of the attack, depressed to a degree in which the immediate or general abstraction of blood might be hazardous, or even of dangerous consequence. In these circumstances, the developement of vascular energy is promoted by immersion in the warm bath, after which venesection is often admissible, and proves highly efficacious in obviating the tendency to congestion. In other cases of this low character of fever, the local abstraction of blood may be advantageously substituted for general depletion; and in a few rare instances of extreme deficiency of vital energy, and torpor, evacuations of blood are to be wholly prohibited, and the cure confided to the warm bath, blisters, and stimuli.

In the chronic structural derangement of some of the abdominal viscera, which is the occasional sequel of the continued yellow-fever, but far more frequently of the recurrent form, and intimately connected with the degree of freedom or limitation with which evacuations have been used in the early stage of the attack, the continued irritation of mercury is eminently serviceable, and commonly successful in the restoration of their functions. A removal to a more northern latitude is, however, not unfrequently essential to permanent recovery.

I have now stated the results of my own experience and observation on the powers of mercury in yellow-fever, unbiassed by that of others. The concurrence of opinion of other observers in different times, and in different situations, must however be considered as the strongest corroboration of the justness of these remarks; and with this intention, I shall append a short review of some of those which occur to me.

Dr Jackson, from extensive experience in St Domingo, admits the mouth is often affected when the disease is mild, or of a remitting type; but " on the contrary, where the disease is continued and ardent, or slow and creeping, with diminished. sensibility of the skin, and impaired energy of the vascular system, enormous quantities of calomel either produce no visible effect, or the gums become spongy and livid, but no salivation ensues: the event is then unfortunate, or life emerged in a gradual manner, Further, it is a common observation, that where 
salivation actually takes place in continued fevers, it seldom shews itself till the violence of the symptoms has evidently abated: hence a suggestion arises, that the appearance of salivation is only an indication of the departure of the disease; - no proof exists, that the operation of the mercury is the cause of this departure."-(Outline of Fever, p. 294.)

Dr liush says, that mercury seldom salivated till the fever intermitted or declined. That the salivation came on during the intermissions, and went off during the exacerbations. (Med. Obs. and Inq. Vol. IV.p. 94.) And that in the city hospital, where it was chiefly depended on, and venesection sparingly used, more than one-half died.-(Ibid. Vol. V. p. 128.)

Dr Grant states, that all who were treated by mercury died, and that they became more victims to the mercury than even to the fever.

Dr Lempriere observes, if considerable relief was not obtained within the first twenty-four hours of the attack, congestions formed in the abdominal viscera and brain, to remove which, mercury was chiefly relied on; but it was found, that immense quantities of calomel were frequently exhibited without exciting any apparent action, owing probably to the torpid state of the absorbents.

Dr Bancroft thinks its utility in yellow-fever, except as a purgative, greatly to be doubted; that it has been extensively tried in the army without success; and that, at any rate, its good effects have been very much exaggerated.-(Essay on Yellow-Fever, p. 76, et seq.)

Even Dr Chisholm allows, that " there are circumstances in which, however, the utmost difficulty is experienced in obtaining this effect (salivation) from calomel, and others in which the candid practitioner must acknowledge its insufficiency."-(Vol. I. p. 253.) And he mentions, that 2000 grains have been given without effect, when under the influence of disease.-(Vol. I. p. 429.)

Sir James Fellowes says, he never saw advantage from the large quantities of calomel recommended in fever.-(Reports, p. 406.)

I shall now proceed to state some of the opinions entertained as to the efficacy of mercury in yellow-fever, more immediately within the period of my own services in the West Indies, by those whose official situations gave them the fullest opportunities of estimating its powers ; premising, that the nature of my review precludes my adverting to a vast weight of oral testimony which I have enjoyed, in communication with the medical officers of both services: 
Dr Dickson, whose opinions on this head I had ample opportunities of knowing, while I was surgeon of the flag-ship on the Leeward Island station, and who, from being furnished officially with the weekly reports of the respective surgeons of the fleet, possessed the most extensive sources of information, conceives that the mercurial influence is incompatible with a great degree of heat and vascular action; and that the most profuse exhibition of this medicine is unable to induce its specific effect during a state of high excitement, as well as in that form of fever, where the sensibility of the system is greatly impaired; where there is time, that is, in protracted instances, where the fever has abated, and the patient, having survived the danger of the earlier stage, labours under symptoms of congestion or organic disease, he is of opinion that mercury is often of the greatest service. But in this first stage of the concentrated endemic, he has generally found every attempt to produce ptyalism to be altogether fruitless, and he therefore concludes, that the cases wherein this has been effected must be referred to a remitting or milder form of disease; and fully coincides with those who are of opinion that salivation " is not the cause, but simply an indication, of the cessation of fever." (Edinburgh Journal, January 1813.)

Dr Macarthur, who necessarily enjoyed ample opportunities of determining the question at Barbadoes, in a report to the naval medical board, which was afterwards published, states that, in the fevers of the crews of the Saint Lucia, and of the Amelia, in 1804, the mercurial practice was pushed to its utmost extent. "The submuriate of mercury," he continues, " in large doses given by the mouth,-mercurial frictions applied to the surface, alternated by the hot and cold baths to induce ptyalism, - were assiduously and perseveringly employed; but, however protracted the fever might have been, no instance occurred where the mouth could be affected in the worst fevers, which terminated in death. In the mild fevers, or where recovery began before the first thirty-six hours, ptyalism was easily induced before the expiration of that time. Many cases of protracted fever have occurred at this hospital, where the mercurial plan was omitted some days before there was any sign of convalescence, but the mercurial action in the mouth did not appear until the fever evidently had ceased, and it increased for some days with the convalescence of the patient. In other cases, where the mouth had been made sore by the mercury exhibited, the patients have had a relapse, and during one night the soreness of the mouth entirely disappeared. It is, therefore, evident, that so long as the morbid actions which 
constitute this fever existed, no mercurial action took place; but as soon as the fever ceased, the mercurial disease commenced. Ptyalism, therefore, was not the cause of the cessation of fever, but the consequence, and the soreness of the mouth only becomes a test of the absence of fever. But the patient pays too dear for this test, if it shall confine him to the hospital, or to his room, for three weeks or a month with a severe salivation, when otherwise, in one week, he would have been able to have returned to his ordinary duty or occupation. Mercury, such as the preparation of the submuriate, has been employed with much advantage as a purgative."

$\mathrm{Mr}$ Mortimer, late surgeon to the Naval Hospital in Barbadoes, in his Official Report on Yellow-Fever, observes, that from three to eight grains of calomel were exhibited by him every third hour at Mariegalante, aided by mercurial frictions, unlimited as to quantity; but in few instances, and in those only as a purge, did it evince any positive effect. The fever subsiding, salivation has come on; but however profuse this became, and, consequently, however protracted the recovery, it afforded no additional security against a future seizure. He farther states, that in all the other public situations which he has held, the only result from calomel, during high febrile action, was as before stated, and that not often; while, on the other hand, he has observed much distress to accompany the ptyalism, without any ultimate advantage: so that he cannot consider it in the light of a remedy, unless when combined with a cathartic. (Med. Chir. Journal, March 1817.)

Lastly, the latest writer on fever, though partial to the use of this medicine in that disease, admits that, "6 while the system continues under the influence of fever, ptyalism is not easily produced." (Armstrong on Typhus, p. 155.) And if such be the case in the comparatively mild and remediable fevers of this climate, some estimate may be formed of its total incompetency in so rapid and uncontrollable a disease as yellow-fever during the inordinate actions of the first stage, the march of which can only be checked by depletion; and, in fine, when only the powers of medicine can be applied with any well-founded hope of success.

Mercury has been recommended as a prophylactic. On this point I do not profess to speak from experience. The rationale of the practice would seem to be grounded on the idea, that whatever cures, necessarily prevents; - an assumption, the fallacy of which I need not expose; for, even were the antecedent true (which I trust has been sufficiently disproved), the consequent would be false. Strict temperance, and a gradual initia. 
tion of the system to those assimilatory changes which the organization of every person exposed to the influence of a tropical climate is destined to endure, will, I believe, be found to afford a more reasonable prospect of security (at least from violent attacks), than the temporary change in the constitution which a saturation with mercury may create.

Witney, Oxfordshire, $2 d$ July, 1817.

\section{IV. .}

Observations on Inflammation and Brain-Fever. By JAMES Wood, M. D. Newcastle.

TN 1793, in a publication on Fever, I remarked, that, in ophI thalmia, we met with inflammation of two kinds, requiring opposite means of cure; the one from debility would not admit of depletion, but the other, from too high action, was capable of being cured both by depletion and stimulants. I observed * that this view in the treatment of the latter, of its being cured by opposite means, had removed my greatest difficulty in the investigation I had begun ; that it solved questions before inexplicable, reconciled apparent contradictions, and gave the most satisfactory explanation of the adage that " doctors differ." It explained how recovery in typhus took place, both under the cooling and stimulating plan of treatment, how both plans were successful in burns and scalds, and, carried to a similar diseased action of the brain, how brain-fever was cured by opposite means. I observed in the same paper, * that, 6 in all such states, I have been invariably successful by the application of cold, with the aid of purgatives; and yet I cannot doubt, from what I have heard and read, that such states have been cured by the use of stimulants. Although, then, common inflammation, typhus fever, brain-fever, and other similar states of diseased action, are capable of being cured by different, and nearly opposite means, yet it does not follow, that both means are equally good, and that one has not the preference to the other. The cooling plan of treatment, or that of directly lessening diseased

In 1808, in a paper in the London Medical Journal, on the treatment of Burns and Scalds, No: 109. 